\title{
A PRELIMINARY STUDY FOR A TETRAHEDRON FORMATION: QUALITY FACTORS AND VISUALIZATION
}

\author{
José J. Guzmán* \\ Conrad Schiff ${ }^{\dagger}$ \\ a.i. solutions, Inc. \\ Mission Design Division \\ 10001 Derekwood Lane, Suite 215 \\ Lanham, MD 20706, USA
}

\begin{abstract}
Spacecraft flying in tetrahedron formations are excellent for electromagnetic and plasma studies. The quality of the science recorded is strongly affected by the tetrahedron evolution. This paper is The quality of the scion on the computation of quality factors and visualization for a formation of four or five satellites. Four of the satellites are arranged geometrically in a tetrahedron shape. If a fifth satellite is present, it is arbitrarily initialized at the geometric center of the tetrahedron. The fifth satellite is present, it is arbitrarily initialized at the geometric center of the tetrahedron. The fith for the initialization. The natural orbit evolution is visualized in geocentric equatorial inertial and in geocentric solar magnetospheric coordinates.
\end{abstract}

\section{INTRODUCTION}

Spacecraft formation flying and/or distributed space systems allow measurements not possible with single spacecraft. For space science missions, observable field parameters (particle populations, electric and magnetic fields) vary both in space and time.' Thus, understanding of the processes within the field requires accurate and precise observations and analysis of both the temporal and spatial variations. A tetrahedron formation is utilized since four spacecraft, with adjustable separations, are the minimum needed to resolve a three-dimensional structure, at least to the lowest order in the physical field gradients. ${ }^{2}$

The appropriate spacecraft separations, of course, depend on the field or event being sampled. As a result, formation flying missions require closer interaction between the project scientists and mission designers than a typical single-spacecraft mission. A means of communicating quantitatively is to define quality factors that affect the data collection and analysis processes. These quality factors are shape- and size-dependent parameters that are functions of the geometric shape and evolution of the formation. Once the scientists have defined the quality factors appropriate for their mission, the mission analysts can try to tailor the trajectory design appropriately. Furthermore, to communicate qualitatively, visualization of

"Mission Analyst, Member AIAA and AAS

$t_{\text {Chief Scientist, Member AAS }}$ the formation - in coordinate frames useful to the scientists is important. This paper is a preliminary study on the computation of quality factors and visualization for a formation of four or five satellites.

\section{TETRAHEDRON MISSIONS}

Scveral missions are planning to use tetrahedron formations for field and plasma studies. In fact, one such mission, Cluster $\mathrm{II}^{*}$, is currently flying and operating successfully. ${ }^{3}$ The Cluster II mission was launched in pairs using Russian Soyuz rockets: two spacecraft launched on July 12, 2000 and two on August 9, 2000. Cluster's primary mission is to explore the SunEarth connection, specifically the interaction of the Sun and the Earth's magnetosphere. In the process of planning the Cluster mission, fuel optimization of the maneuvers needed to initialize, modify and maintain the formation was considered by J. RodríguezCanabal and M. Belló-Mora. ${ }^{4}$ Later a different optimization method was employed by J. Schoenmaekers. ${ }^{5}$ In J. Schoenmaekers's paper, both methods are compared for one mission scenario. Moreover, a furtherdeveloped strategy is presented by M. Bello-Mora and Rodríguez-Canabal. ${ }^{6}$ Implementation and operational results for the maneuvers are reported by D. Hockens and J. Schoenmaekers. ${ }^{7}$

*Cluster II is a replacement of the original Cluster mission which was lost in a launch failure during the maiden flight of the Ariane 5 rocket on June 4, 1996. 
The Auroral Lites mission concept also proposed using four spacecraft. This mission's main goal would have been to acquire an unprecedented understanding of the multiscale dynamics of space plasmas by exploring the Earth's auroral zone. ${ }^{8,9}$ Auroral Lites also would have been a technology pathfinder for flying formations of small spacecraft. Unfortunately, the mission did not make the final selection of the $1998 \mathrm{NASA}$ Medium-class Explorers (MIDEX) program. Another proposed mission that will utilize a tetrahedron formation is the Magnetospheric Multiscale (MMS) Mission. ${ }^{10}$ MMS will determine the small-scale basic plasma processes which transport, accelerate and energize plasmas in thin boundary and current layers. These processes control the structure and dynamics of the Earth's magnetosphere. Preliminary mission design and analysis for the MMS reference path, which includes a double lunar swingby for one of its phases, has been presented by A. Edery and C. Schiff. ${ }^{11}$

\section{THE VOLUMETRIC TENSOR}

The spatial gradient of a field observed with multiple spacecraft is dependent on the inverse of a symmetric tensor formed from the positions of the spacecraft (see Harvey"). Harvey adds that "the importance of the volumetric tensor for describing the geometry of a tetrahedron was first noted by J. Schoenmaekers of the European Space Operations Centre (ESOC) Flight Dynamics Division". Thus, this tensor is important for both the gradient calculations and for providing information about the shape and orientation of the formation. For $N$ spacecraft, in an arbitrary frame $I$ and about the mesocenter (mean position or centroid), the volumetric tensor can be expressed as,

$$
\overline{\bar{R}}^{I / m c}=\frac{1}{N} \sum_{i=1}^{N} \bar{r}_{m c i} \bar{r}_{m c i}^{T},
$$

where the double bar is used to indicate a second order tensor, and $\bar{r}_{m i}$ is the position (column) vector of the $i$ th spacecraft relative to the mesocenter, $m c$. Relative to the origin of the arbitrary frame $I$, the mesocenter is computed as,

$$
\vec{r}_{m c}=\frac{1}{N} \sum_{i=1}^{N} \bar{r}_{i},
$$

where $\bar{r}_{i}$ is the position vector of the $i$-th spacecraft relative to the origin of the arbitrary frame $I$. The mesocenter is equal to the system's center of mass if all the spacecraft have the same mass. Furthermore, if the tetrahedron is regular (the separations between vertices are equal), the mesocenter equals the geometric center (a point equidistant from all vertices). For a tetrahedron, it can be shown that the volume $V$ is given by,

$$
V=\frac{8}{3} \sqrt{\mid \overline{\bar{R}}^{I / m c \mid}}
$$

where $\left|\overline{\bar{R}}^{I / m c}\right|$ is the determinant of the tensor. Hence the name, volumetric tensor.

\section{Relationship to the Inertia Tensor}

The inertia tensor about the mesocenter is closely related to the volumetric tensor. In fact, the eigenvectors are the same and the eigenvalue associated with the largest (smallest) eigenvalue of the inertia tensor is associated with the smallest (largest) eigenvalue of the volumetric tensor. See Harvey ${ }^{1}$ for the equation relating the eigenvalues.

\section{Pseudo-Ellipsoid}

Analogous to the inertia-ellipsoid, a pseudo-ellipsoid can be constructed with the eigenvalues and eigenvectors of the volumetric tensor. Therefore, the pseudoellipsoid has information about the shape and orientation of the formation. This proves very useful in the formulation of quality factors.

\section{NATURAL COORDINATES}

For both mission design and science purposes, it might be convenient to sometimes work in a coordinate frame that is dependent on the shape of the tetrahedron. This can be accomplished by utilizing natural coordinates. Natural coordinates are typically used in finite element analysis. ${ }^{12}$ Analogous to the volumetric tensor, the tetrahedron natural coordinates are dependent on the volume of the tetrahedron. A tetrahedron in rectangular and natural coordinates is displayed in Figure 1. The faces of the tetrahedron are numbered

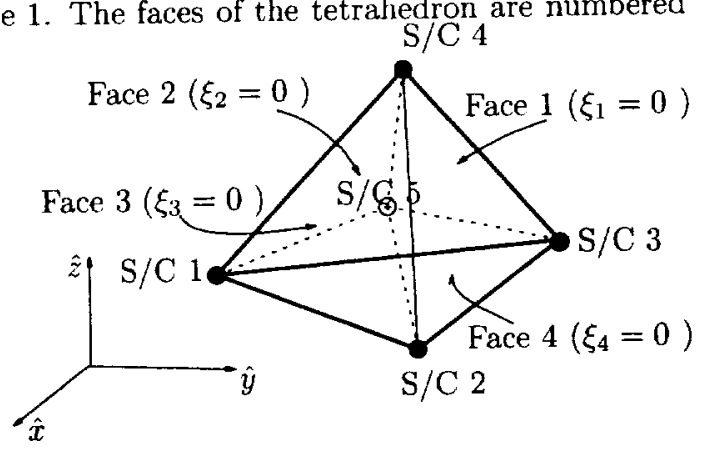

Fig. 1 Tetrahedron Natural Coordinates

such that the $i$-th face is opposite to the $i$-th vertex (spacecraft). The tetrahedral coordinates for an interior point, $\bar{\xi}=\left\{\xi_{1}, \xi_{2}, \xi_{3}, \xi_{4}\right\}^{T}$, are defined as the ratio of the volume of the subtetrahedron formed by the interior point and face $i$ to the tetrahedron volume. That is, $\bar{\xi}=\left\{V_{1} / V, V_{2} / V, V_{3} / V, V_{4} / V\right\}^{T}$. Thus, the $i$-th face has $\xi_{i}=0$. Furthermore, note that

$$
V_{1}+V_{2}+V_{3}+V_{4}=V
$$

which implies that,

$$
\xi_{1}+\xi_{2}+\xi_{3}+\xi_{4}=1
$$

Therefore, with this last equation and relating the cartesian coordinates $\{x, y, z\}$ of an interior point to

2 


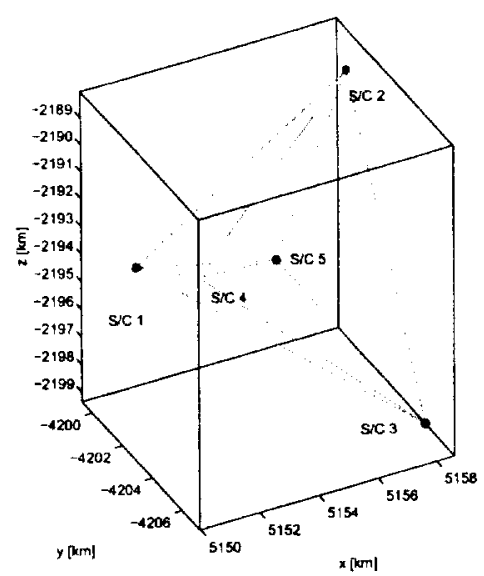

Fig. 2 Example: Inertial Coordinates

the tetrahedral coordinates,

$$
\left\{\begin{array}{l}
1 \\
x \\
y \\
z
\end{array}\right\}=\left[\begin{array}{cccc}
1 & 1 & 1 & 1 \\
x_{1} & x_{2} & x_{3} & x_{4} \\
y_{1} & y_{2} & y_{3} & y_{4} \\
z_{1} & z_{2} & z_{3} & z_{4}
\end{array}\right]\left\{\begin{array}{l}
\xi_{1} \\
\xi_{2} \\
\xi_{3} \\
\xi_{4}
\end{array}\right\}
$$

where the cartesian coordinates of the points that define the tetrahedron are given by the vectors $\bar{r}_{i}=$ $\left\{x_{i}, y_{i}, z_{i}\right\}^{T}$ for $i=1, \ldots, 4$. For example, in natural coordinates the geometric center of a regular tetrahedron is located at $\bar{\xi}=\{1 / 4,1 / 4,1 / 4,1 / 4\}^{T}$. Then, using Equation (6) the geometric center cartesian coordinates are readily available. See Figure 2 for an example with orbital data. The transformation from cartesian to natural coordinates is given by taking the inverse of the matrix in Equation (6), provided the tetrahedron volume is non-zero.

\section{Aside: Volume Integrals}

It should be noted that natural coordinates simplify the integration over the volume of a tetrahedron. This may be useful for some of the science analysis. In fact, the integration limits for each variable become constants. For example, for an integral, $\kappa$, of a function, $f$, over the volume of the tetrahedron,

$$
\begin{aligned}
\kappa & =\int_{\mathrm{Vol}} f(x, y, z) d V \\
& =\int_{0}^{1} \int_{0}^{1-\xi_{1}} \int_{0}^{1-\xi_{1}-\xi_{2}} f\left(\xi_{1}, \xi_{2}, \xi_{3}, \xi_{4}\right) d \xi_{3} d \xi_{2} d \xi_{1} .
\end{aligned}
$$

As a result, the integrations may be easily obtained numerically and even exactly. See Yang ${ }^{12}$ for details and examples.

\section{QUALITY FACTORS}

The quality factors are shape- and size-dependent parameters that affect the resolution of spatial gradients. Many have been examined and compared, but in this paper the ones examined by $P$. Robert et al. ${ }^{13}$ are utilized. In general, the quality factors can aid in defining the formation geometry and stationkeeping requirements. Before introducing some of them, various parameter definitions are required.

\section{Some Geometric Definitions}

First, recall that in a regular tetrahedron the separations between vertices are equal. Then, the ideal volume and surface are defined as the volume and surface area of a regular tetrahedron with vertex separations equal to the average of the 6 distances between the true, or actual, vertices. The circumscribing sphere is defined as the sphere with center at the geometric center and radius equal to the distance from that center to any spacecraft (thus, all 4 spacecraft lie on its surface).

\section{One-Dimensional Parameters}

The following parameters are useful for comparing the true tetrahedron against an ideal (regular) tetrahedron. A regular tetrahedron might be useful in cases where sampling of the structure of a field is not as important as understanding its transient or fluctuating events. $^{13}$

- The Glassmeier $Q_{\mathrm{GM}}$ parameter is defined as,

$$
Q_{\mathrm{GM}}=\frac{\text { True Volume }}{\text { Ideal Volume }}+\frac{\text { True Surface }}{\text { Ideal Surface }}+1 \text {. }
$$

This parameter takes values between 1 (the four spacecraft are in a line) and 3 (a regular tetrahedron).

- The Robert/Roux $Q_{\mathrm{RR}}$ parameter is defined as,

$$
Q_{\mathrm{R} R}=\left(\frac{9 \pi}{2 \sqrt{3}} \cdot \frac{\text { True Volume }}{\text { Sphere Volume }}\right)^{\frac{1}{3}},
$$

where the sphere volume is the volume of the circumscribing sphere. This parameter has a minimum value of 0 and equals 1 for a regular tetrahedron.

- The $Q_{\text {sr }}$ parameter is defined as,

$$
Q_{\mathrm{SR}}=\frac{1}{2}\left(\frac{\tilde{a}+\tilde{b}+\tilde{c}}{\tilde{a}}-1\right) .
$$

This parameter uses information from the volumetric tensor, namely its eigenvalues. Specifically, referring to the volumetric tensor, let $\tilde{a}, \tilde{b}, \tilde{c}$ be its largest, middle and smallest eigenvalues respectively.

- The $Q_{\mathrm{R} s}$ parameter is defined as,

$$
Q_{\mathrm{Rs}}=\frac{\text { True Volume }}{\text { Ideal Volume }} \text {. }
$$

This parameter is the first term of Equation (7). It has a minimum value of zero and equals 1 for a regular tetrahedron. 


\section{Two-Dimensional Parameter Space}

Now, in an effort to have some parameters that describe the shape of the formation better, a twodimensional parameter space can be defined by utilizing the concepts of elongation (prolateness) and planarity (oblateness) of the pseudo-ellipsoid. Following P. Robert et al., ${ }^{13}$ the elongation and planarity are computed as,

$$
\begin{aligned}
& \tilde{E}=1-(\tilde{b} / \tilde{a}) \\
& \tilde{P}=1-(\tilde{c} / \tilde{b}) .
\end{aligned}
$$

Different characteristic shapes can de defined in terms of the values of $\tilde{E}$ and $\tilde{P}$. Both parameters take values between 0 and 1 . In fact, if $\tilde{E}=\tilde{P}=0$, the tetrahedron is regular; if $\tilde{E}=1$ the four spacecraft are co-linear; and, if $\tilde{P}=1$ the four spacecraft are co-planar. ${ }^{13}$

\section{SCIENTIFIC COORDINATE FRAMES}

Several coordinate frames are useful for electromagnetic and plasma studies. ${ }^{14}$ Thus, these frames are important for visualization of the satellite formation in a frame relevant to the scientists. For now, two frames are considered: (1) the Solar Magnetospheric (GSM) coordinate system, and (2) the Solar Magnetic (SM) coordinate system. These frames are defined as follows. First, let $\hat{r}_{\oplus \mathcal{O}}$ be a unit vector in the Earth to Sun direction ${ }^{\dagger}$ and $\hat{n}_{d}$ be a unit vector in the inagnetic north direction. $\ddagger$ Then, the two frames of interest are defined, in terms of the axis directions, as

1. GSM coordinate system: $\hat{X}_{\mathrm{GSM}}=\hat{r}_{\oplus 0}, \hat{Y}_{\mathrm{GSM}}=$ $\frac{\hat{n}_{d} \times \dot{r}_{\oplus 0}}{\left\|\hat{n}_{\mathrm{d}} \times \hat{r}_{\oplus \mathrm{e}}\right\|}, \hat{Z}_{\mathrm{GSM}}=\hat{X}_{\mathrm{GSM}} \times \hat{Y}_{\mathrm{GSM}}$, and

2. SM coordinate system: $\hat{Z}_{\mathrm{SM}}=\hat{n}_{d}, \hat{Y}_{\mathrm{SM}}=$ $\frac{\hat{n}_{d} \times \hat{r}_{\oplus \mathcal{O}}}{\left|\hat{n}_{d} \times \hat{r}_{\oplus \Theta}\right|}, \hat{X}_{\mathrm{SM}}=\hat{Y}_{\mathrm{SM}} \times \hat{Z}_{\mathrm{SM}}$.

In both cases, the Earth dipole axis is in the $\hat{X}-\hat{Z}$ plane. The angle between the north magnetic pole, $\hat{n}_{d}$, and the $\hat{Z}_{\mathrm{GSM}}$ axis is called the dipole tilt angle. Thus, the difference between these two frames is a rotation about the $\hat{Y}$ axes (which are the same for both frames). The rotation angle is simply the dipole tilt angle. In this paper, only the GSM tetrahedron evolution is shown.

t The Earth to Sun vector is computed using either ephemeris or an approximation.

$\ddagger$ The dipole axis is computed using dipole angles from the International Geomagnetic Reference Field (IGRF). The IGRF is a series of mathernatical models of the Earth's main magnetic field and its secular variation. Each model comprises a set of spherical harmonic (or Gauss) coefficients in a series expansion of the geomagnetic potential. ${ }^{15}$

\section{APPROACH}

To test all the concepts introduced, several runs were performed. Specifically, the quality factors are computed for the tetrahedrons in Table 1. The parameters associated with these test cases are discussed next.

\begin{tabular}{|c|c|}
\hline \hline Formation Number & Tetrahedron Spacecraft \\
\hline \hline 1 & $(1,2,3,4)$ \\
2 & $(1,2,3,5)$ \\
3 & $(1,2,4,5)$ \\
4 & $(1,3,4,5)$ \\
5 & $(2,3,4,5)$ \\
\hline
\end{tabular}

Table 1 Tetrahedron Permutations

\section{Software}

For this study several pieces of software are utilized: FreeFlyer ${ }^{16}$ MATLAB ${ }^{\text {( }}$ and some internal legacy $\mathrm{C}$ code that transforms to and from the inertial and scientific frames. FreeFlyer ${ }^{\otimes}$ has an embedded scripting language with many capabilities, including calling $M A T L A B^{\oplus}$ and other programs (calls can be made in both directions). In this case, the legacy $\mathrm{C}$ code is compiled as a MATLAB $B^{\circledast}$ MEX file that FreeFlyer ${ }^{\circledast}$ can then access.

\section{Force Model}

All spacecraft are assumed to have the same physical properties: a dry mass of $200 \mathrm{~kg}$, an area of 0.6613 $\mathrm{m}^{2}$, a drag coefficient of 2.2 , and a 1.4 reflectivity coefficient. The FreeFlyer ${ }^{\circledR}$ integrator selected uses the Runge-Kutta-Verner $8(9)$ formulas. ${ }^{17}$ The force nıodel selected has the following contributions: $8 \times 8$ Earth potential model, atmospheric drag, solar radiation pressure, and the Sun and the Moon as point masses.

\section{Reference Orbit}

The reference orbit is selected to be the same as in Hametz et al. ${ }^{9}$ For scientific reasons, it is designed as a $500 \times 7000 \mathrm{~km}$ orbit at an Earth equatorial inclination of 80 degrees. The elements used to define the initial state are in $J_{2}$ Brouwer-Lyddane elements (BLJ2).

\section{Initializing the Formation}

A regular tetrahedron is utilized as the shape required to meet the science objectives. An initial separation of $10 \mathrm{~km}$ is used. Recall that a regular tetrahedron might be useful in cases where understanding transient and fluctuating events ${ }^{13}$ is important. The initial formation geometry is specified via the FreeFlyer ${ }^{\circledast}$ close formation utility. In this utility, a reference orbit is specified and the individual spacecraft locations are specified in the (rotating) velocity-binormal-normal (VBN) frame. Specifically, each spacecraft location is specified using spherical coordinates in the VBN frame. ${ }^{18}$ In terms of velocity 
the velocity direction is kept the same as that of the reference orbit, i.e. the VBN frame velocity direction. Then, the velocity magnitude is obtained using the two-body problem (2BP) energy equation, thus, $v=\sqrt{\mu(2 / r-1 / a)}$, where $v$ is the velocity magnitude, $r$ is the position magnitude (radial distance), $\mu$ is the gravitational parameter and $a$ is the semi-major axis.

The fifth spacecraft is initialized to be at the geometric center of the formation. (This is easily done by utilizing tetrahedron natural coordinates). It is assumed that these initial conditions can be achieved by the spacecraft from some initial orbit(s). Of course, different initial conditions should be tried to lower the fuel cost to initialize the formation. This optimization process will depend on the pre-initialization orbital states which in turn depend on the launch to orbit strategy. Examples of formation initialization are discussed by Mailhe et al. ${ }^{19}$ and by Hughes and Mailhe. ${ }^{20}$

\section{A Note on Stationkeeping and Relative Orbits}

In this paper the formation stationkeeping and/or re-configuration problem is not considered. Nevertheless, many efforts are in progress in this arena for different types of missions. See for instance the work by Chao et al. ${ }^{21}$ In terms of relative orbit design, the paper by Alfriend and Schaub provides a good summary and some possible solutions for Earth orbiting platforms. ${ }^{22}$ It should be noted that for close formations and/or precise formations, relative navigation and communication is most likely needed.

\section{RESULTS}

Plots of quality factors as a function of BLJ2 mean anomaly for one orbit and for the 5 tetrahedron permutations (Table 1) are displayed in Figures 3-6. The tetrahedron is initialized at 4 different points corresponding to mean anomalies of 0 (Figure 3), 90 (Figure 4), 180 (Figure 5) and 270 degrees (Figure 6). It appears that switching from one formation permutation to another might allow the maximization of a particular quality factor. This would allow taking advantage of the natural dynamics and should be further investigated. In general, the location along the orbit chosen for the formation initialization should represent the region where the primary science will be carried out. Thus, for space science missions, it is possible to have two formation control regimes. The first one would be a "no-science control" regime where the goal is to maintain the spacecraft within a certain maximum separation that will facilitate a smooth transition into the "science control" regime where the formation geometry and/or quality factors is enforced to the required (and feasible) tolerances.

\section{Keplerian Turning Points}

In most of the quality factor figures, note the presence of at least 5 turning points. These points take place at true anomalies of $0,90,180,270$ and back to 0 . (The plots are with respect to BLJ2 Mean Anomaly, thus, in this case, the points are at mean anomalies of approximately $0,54,180,306$ and back to 0 ). The presence of the turning points is not surprising since, from the $2 \mathrm{BP}$, a Keplerian orbit has a radial distance rate given by

$$
\dot{r}=\frac{e h \sin \left(\theta^{*}\right)}{p},
$$

where $e$ is the orbit eccentricity, $h=\bar{r} \times \dot{\vec{r}}$ is the specific angular momentum, $\theta^{*}$ is the true anomaly and $p=a\left(1-e^{2}\right)$ is the semi-latus rectum. Thus, the maximum and minimum radial rate changes occur at true anomalies of 90 and 270 degrees, respectively; and the radial rate is 0 at the apses (true anomalies of 0 and 180 degrees).

The geometry of the formation (initialized at a BLJ2 mean anomaly of 180 degrees or at apogee) is shown at the 5 turning points in Figures 7 and 8 in the GSM frame. For this particular initialization, note that for true anomalies of 270 and 90 degrees, the 4 th and 5 th spacecraft appear to be on top of each other! In fact, the distance between these spacecraft at these two points is about 14 and 20 meters respectively. This case, therefore, would need some type of active control to maintain the 5th spacecraft at a safe distance.

\section{SUMMARY}

This study was an effort to compute the quality factors associated with tetrahedron formations and to visualize the geometric evolution of the formation. Tetrahedron permutations were examined by considering a formation of four satellites and adding a fifth satellite. The fifth satellite was placed at the geometric center of the tetrahedron chosen for the first four satellites. The concept of the volumetric tensor and quality factors were reviewed and implemented. Also, tetrahedron natural coordinates were introduced. These coordinates might be very useful for further studies. Other initial conditions for the fifth satellite and switching from one formation permutation to another in order to maximize a particular quality factor should be investigated. In general, the location along the orbit chosen for the formation initialization should represent the region where the primary science will be carried out. Of course, this location will affect the quality factors. Also, varying the initial distances in the formation should be studied. It is of interest to know how the science is affected by the evolution of the tetrahedron, a key factor in defining the stationkeeping requirements. Furthermore, the quality factors could be used in a feedback mechanism to control the formation shape.

\section{ACKNOWLEDGEMENTS}

Part of this work was done under NASA contract NASS-01090/Task 115. Special thanks to Dr. Steven 
A. Curtis and Mr. David C. Folta from NASA Goddard Space Flight Center.

\section{REFERENCES}

${ }^{1}$ C. Harvey, "Spatial Gradients and the Volumetric Tensor," Analysis Methods for Multi-Spacecraft Data (G. Paschmann and P. Daly, eds.), pp. 307-322, Noordwijk, The Netherlands: ISSI Report SR-001, ESA Publications Division, 1998.

${ }^{2}$ G. Paschmann and P. Daly, "Introduction," Analysis Methods for Multi-Spacecraft Data (G. Paschmann and P. Daly, eds.), Noordwijk, The Netherlands: ISSI Report SR-001, ESA Publications Division, 1998.

${ }^{3}$ R. Mugellesi-Dow, J. Dow, and G. Gienger, "Cluster II: From Launch to First Constellation," 16th International Symposium on Space Flight Dynamics, Pasadena, California, December 2001.

${ }^{4} \mathrm{~J}$. Rodríguez-Canabal and M. Belló-Mora, "Cluster: Consolidated Report on Mission Analysis," European Space Operation Centre, Technical Report, Darmstadt, Germany, July 1990. ESOC CL-ESC-RP-0001.

${ }^{5} \mathrm{~J}$. Schoenmaekers, "Cluster: Fuel Optimum Spacecraft Formation Control," ESA Symposium on Space Flight Dynam ics, Darmstadt, Germany, pp. 419-425, European Space Agency, December 1991. SP-326.

${ }^{6} \mathrm{M}$. Belló-Mora and J. Rodriguez-Canabal, "On the 3-d Configurations of the Cluster Mission," ESA Symposium on Space Flight Dynamics, Darmstadt, Germany, pp. 471-479, European Space Agency, December 1991. SP-326.

${ }^{7} \mathrm{D}$. Hocken and $\mathbf{J}$. Schoenmaekers, "Optimization of Cluster Constellation Manoeuvres," 16th International Symposium on Space Flight Dynamics, Pasadena, California, December 2001.

${ }^{8} \mathrm{~S}$. Curtis, "Auroral Lites, A MIDEX International Multiprobe Mission Proposal," NASA Goddard Space Flight Center, Greenbelt, Maryland, August 1998.

${ }^{9} \mathrm{M}$. Hametz, D. Conway, and K. Richon, "Design of a Formation of Earth Orbiting Satellites: The Auroral Lites Mission," Praceedings of the 1999 NASA GSFC Flight Mechanics and Estimation Conference, Greenbelt, Maryland, pp. 295-308, 1999.

${ }^{10} \mathrm{~S}$. Curtis, "The Magnetospheric Multiscale Mission...Resolving Fundamental Processes in Space Plasmas," NASA Goddard Space Flight Center, Greenbelt, Maryland, December 1999. NASA/TM-2000-209883.

${ }^{11}$ A. Edery and C. Schiff, "The Double Lunar Swingby of the MMS Mission," 16th International Symposium on Space Flight Dynamics, Pasadena, California, December 2001.

${ }^{12} \mathrm{~T}$. Yang, Finite Element Structural Analysis. New Jersey: Prentice-Hall, Inc., 1986.

${ }^{13}$ P. Robert, A. Roux, C. Harvey, M. Dunlop, P. Daly, and K. Glassmeier, "Tetrahedron Geometric Factors," Analysis Methods for Multi-Spacecraft Data (G. Paschmann and P. Daly, eds.), pp. 323-348, Noordwijk, The Netherlands: ISSI Report SR-001, ESA Publications Division, 1998.

${ }^{14} \mathrm{C}$. Russell, "Geophysical Coordinate Transformations," Cosmic Electrodynamics, No. 2, 1971, pp. 184-196.

${ }^{15} \mathrm{C}$. Barton, "Revision of International Geomagnetic Reference Field Released," http://ww .agu.org/oos_elec/95242e. html, 1996. American Geophysical Union.

${ }^{16} \mathrm{M}$. Hametz, D. Conway, and D. Weidow, "New Tools for Formation Monitoring and Maintenance," 1999 American Control Conference, San Diego, California, June 2-4 1999.

${ }^{17} \mathrm{~J}$. Verner, "Explicit Runge-Kutta Methods with Estimates of the Local Truncation Error," Society for Industrial and Applied Mathematics (SIAM) Journal of Numerical Analysis, Vol. 15, August 1978, pp. 772-790.

${ }^{18} \mathrm{C}$. Schiff, D. Rohrbaugh, and J. Bristow, "Formation Flying in Elliptical Orbits," 2000 IEEE Aerospace Conference, Big Sky, Montana, April 18-25 2000. Paper 317.
${ }^{19}$ L. Mailhe, C. Schiff, and S. Hughes, "Formation Flying in Highly Elliptical Orbits: Initializing the Formation," Proceedings of the International Symposium on Space Dynamics, Biarritz, France, CNES, June 26-30 2000. Paper MSO0/21.

${ }^{20}$ S. Hughes and L. Mailhe, "A Preliminary Formation Flying Orbit Dynamics Analysis for Leonardo-BRDF," 2001 IEEE Aerospace Conference, Big Sky, Montana, March 10-17 2001. Paper 905.

${ }^{21}$ C. Chao, J. Pollard, and S. Janson, "Dynamics and Control of Cluster Orbits for Distributed Space Missions," AAS/AIAA Spacefight Mechanics Meeting, Breckenridge, Colorado, February 7-10 1999. AAS 99-126.

${ }^{22} \mathrm{~K}$. Alfriend and H. Schaub, "Dynamic and Control of Spacecraft Formations: Challenges and Some Solutions," Journal of the Astronautical Sciences, Vol. 48, April-September 2000 , pp. $249-267$. 


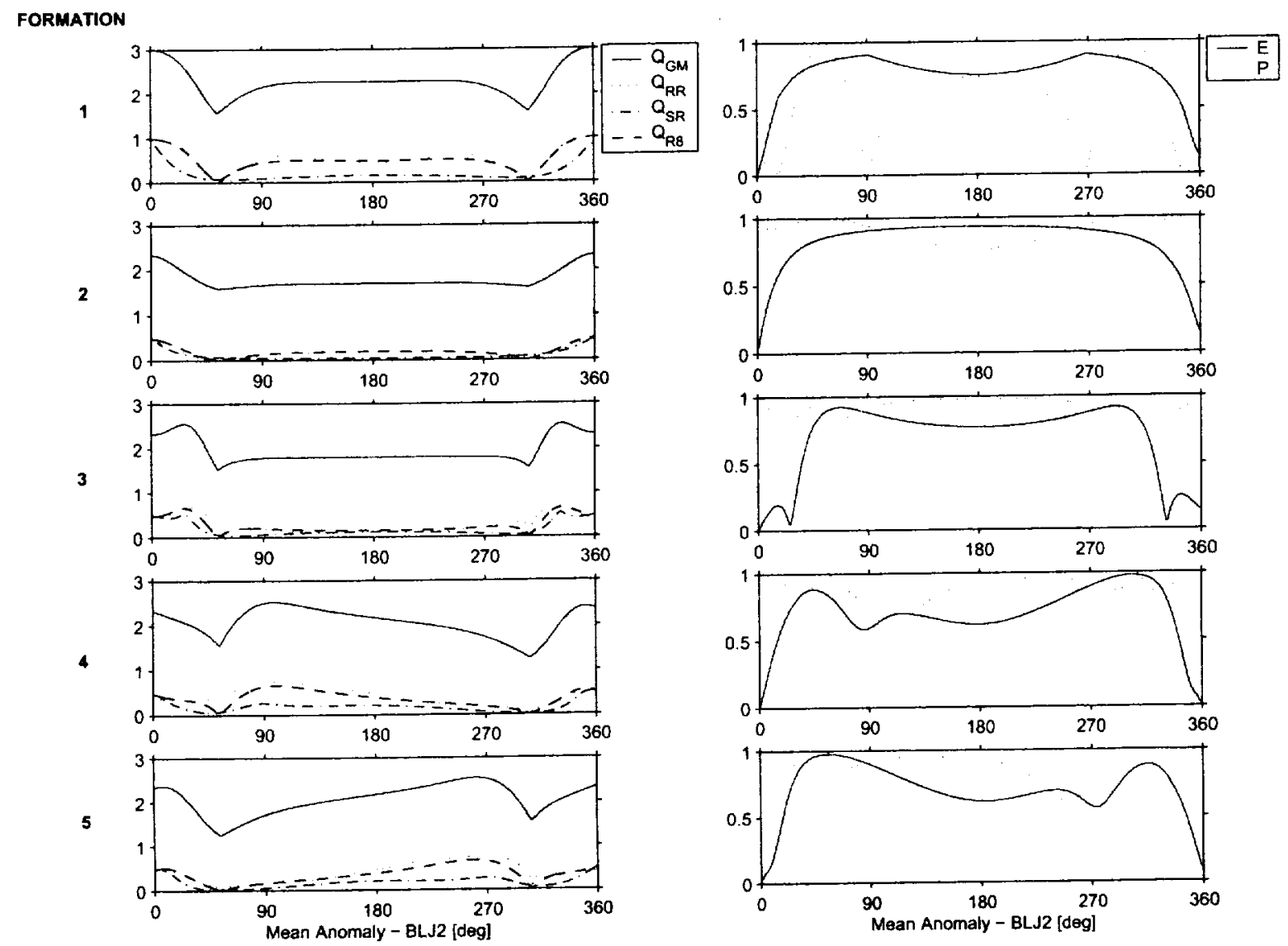

Fig. 3 Initializing at BLJ2 Mean Anomaly of 0 degrees 

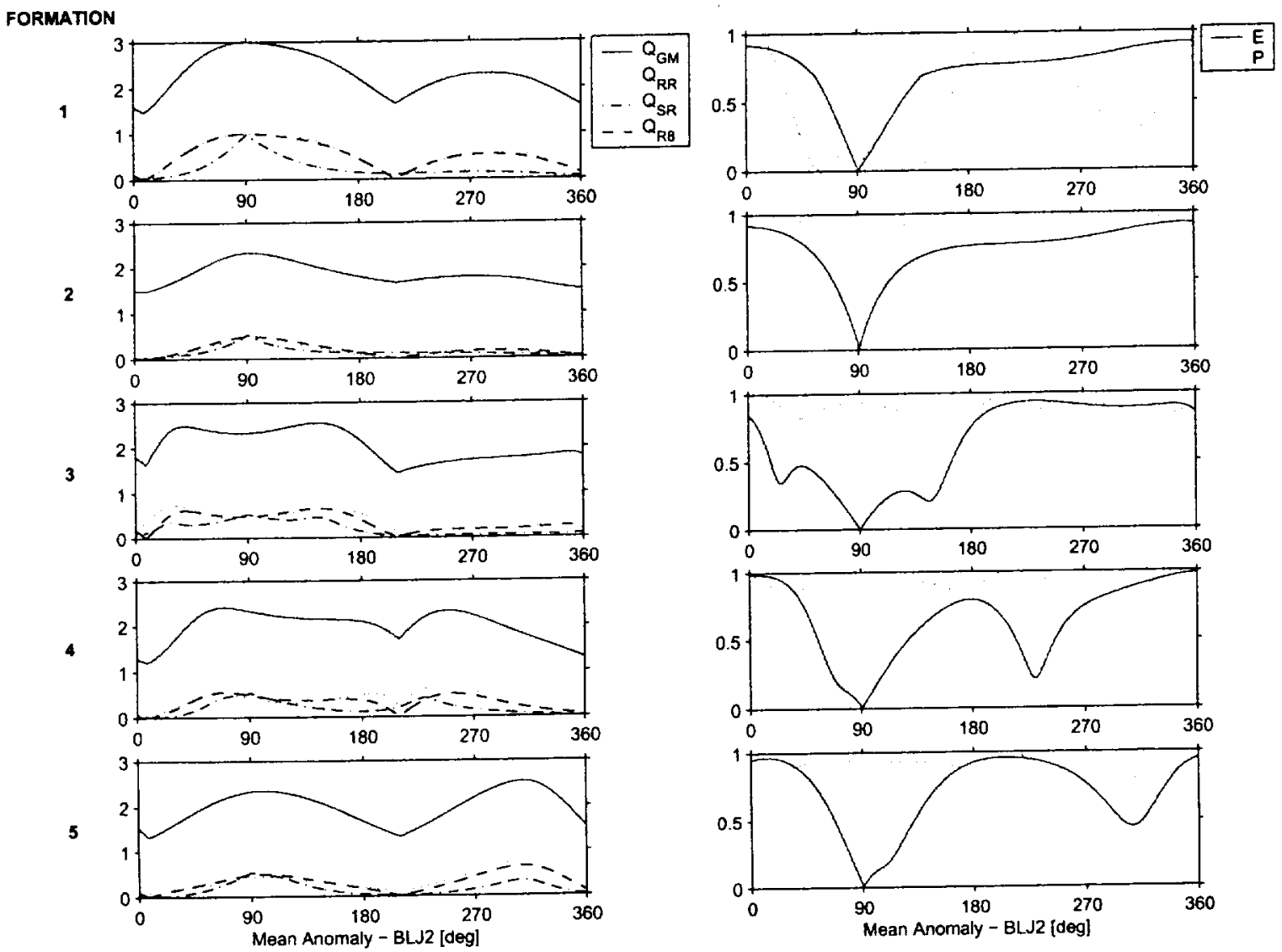

Fig. 4 Initializing at BLJ2 Mean Anomaly of 90 degrees 


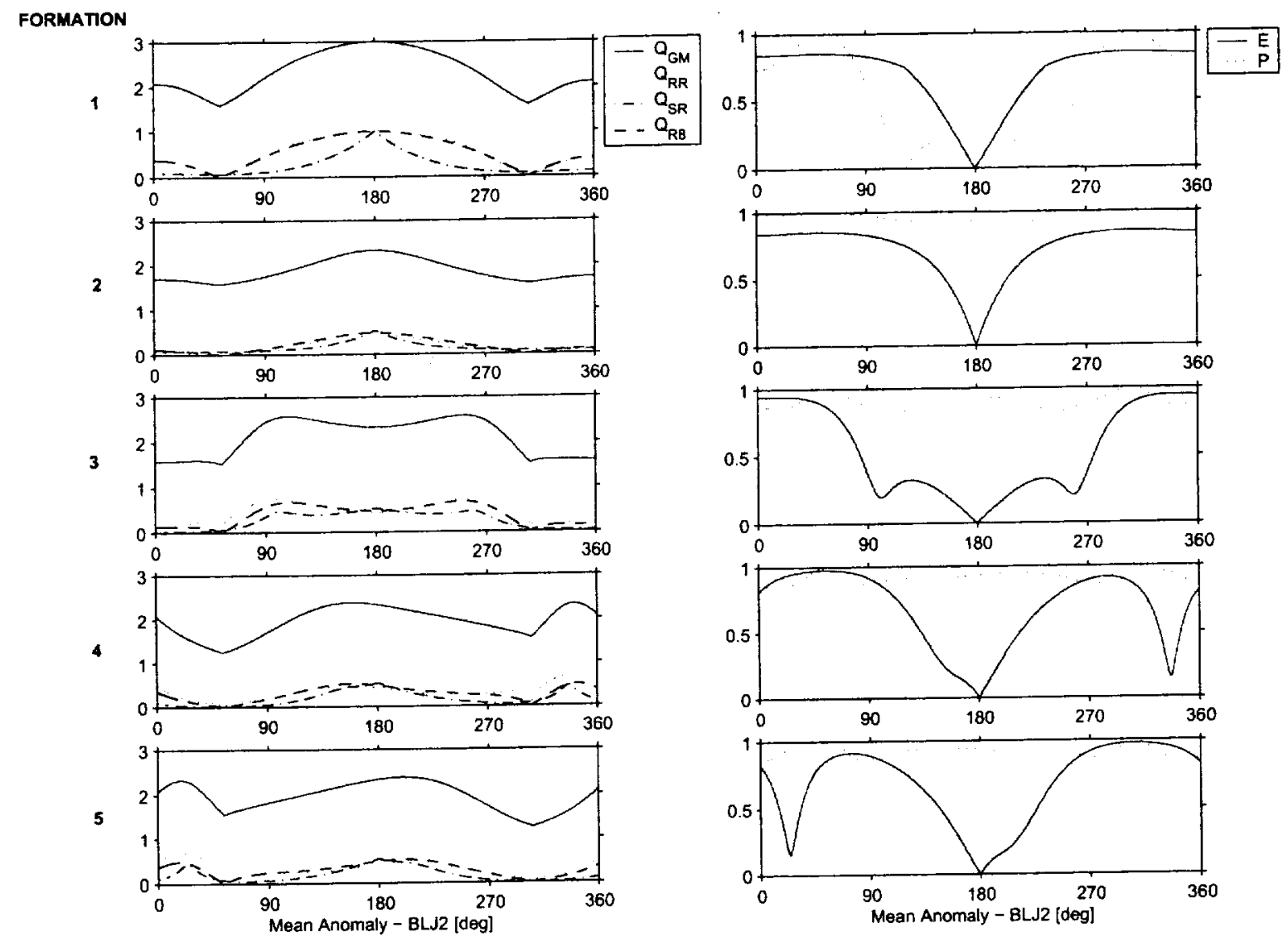

Fig. 5 Initializing at BLJ2 Mean Anomaly of 180 degrees 


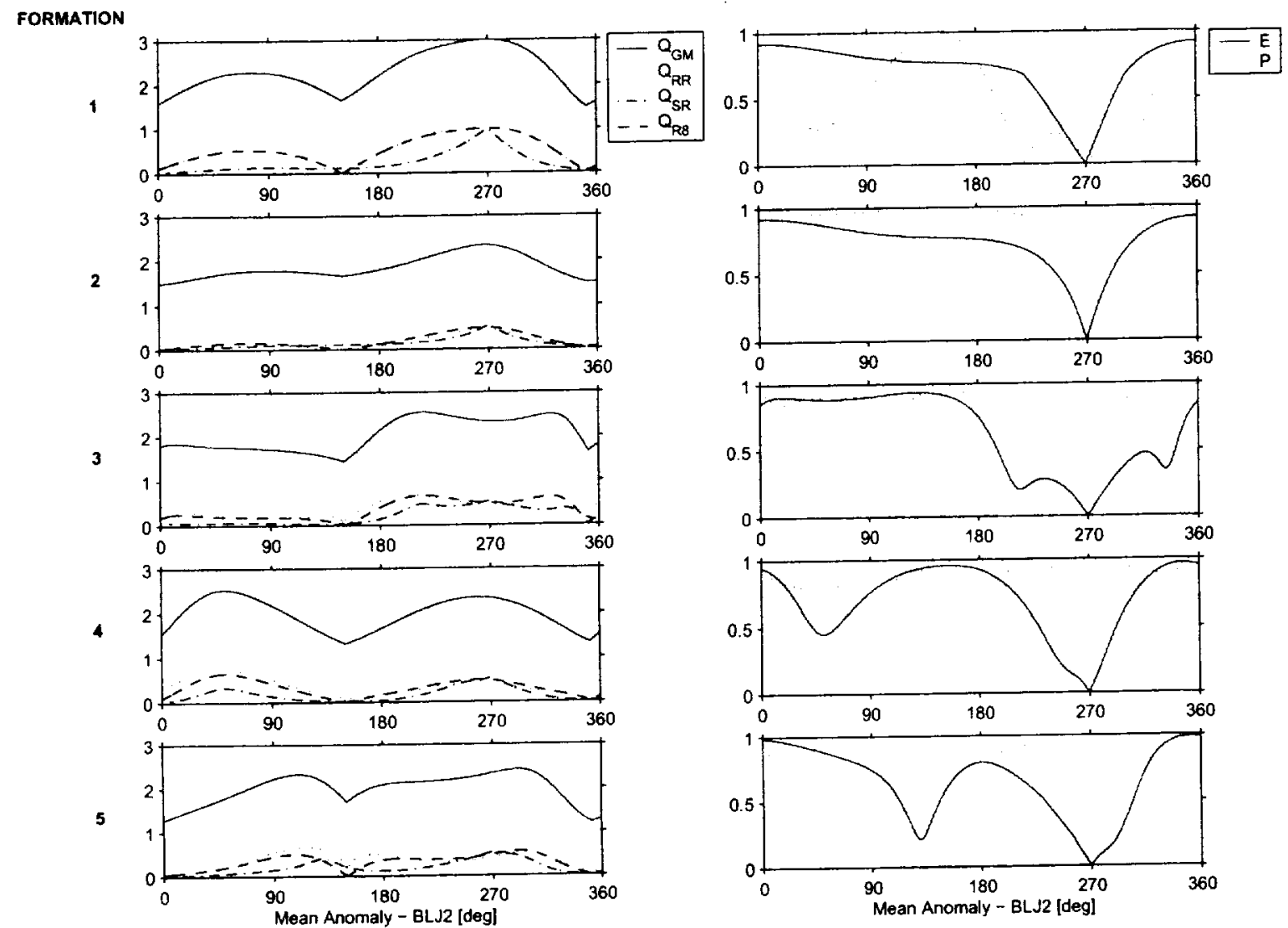

Fig. 6 Initializing at BLJ2 Mean Anomaly of 270 degrees 


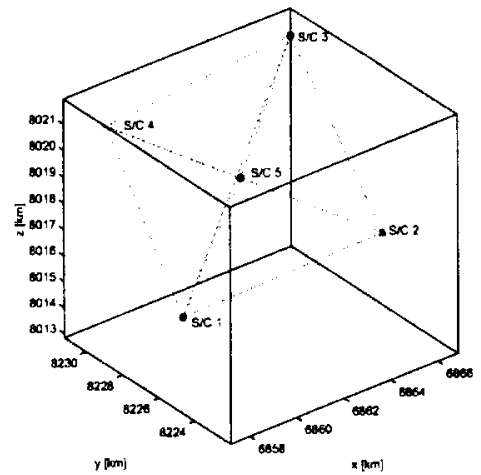

Fig. 7 GSM Initial Formation; TA $=180 \mathrm{deg}$
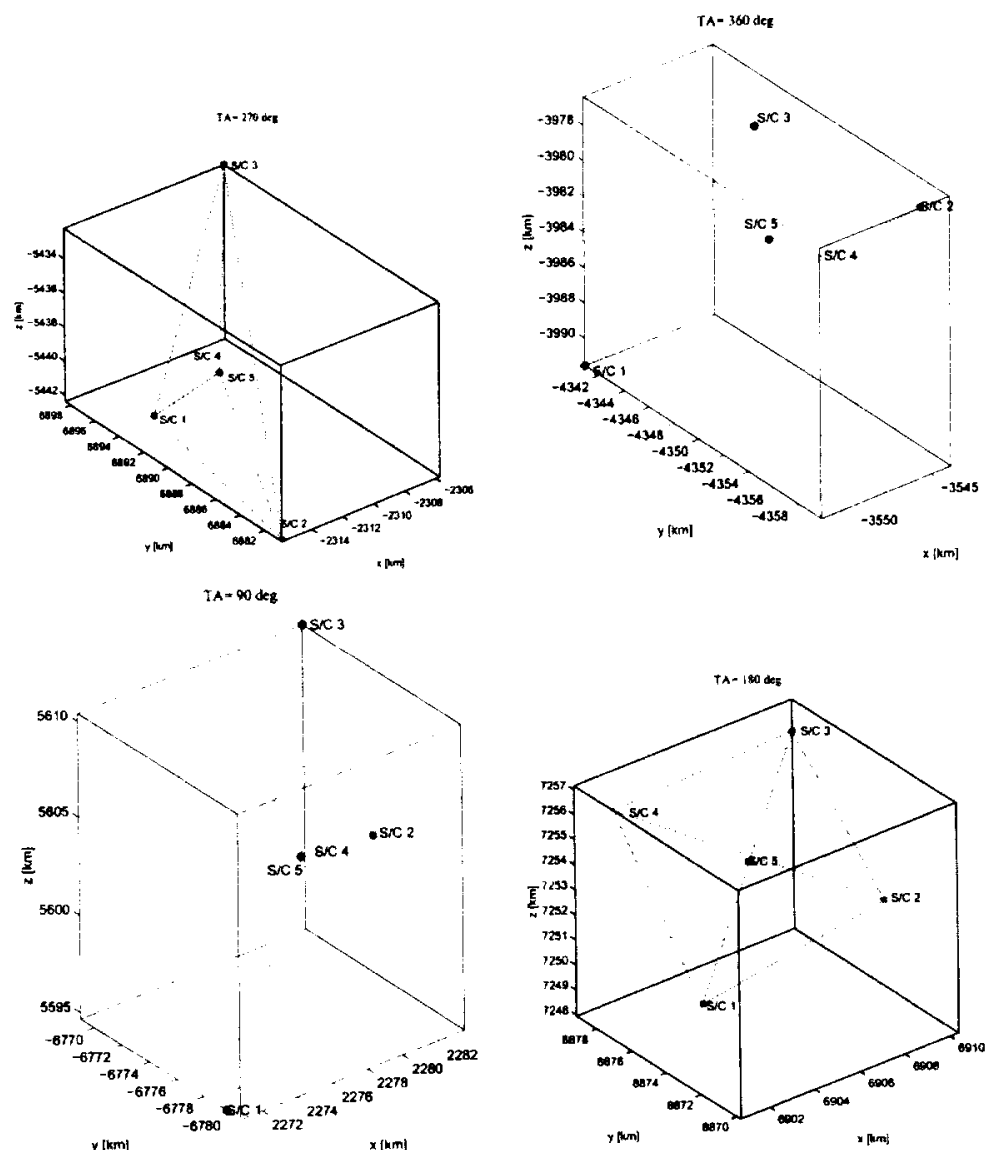

Fig. 8 GSM Formation Evolution; TA= 270, 360, 90, and back to $180 \mathrm{deg}$ (left to right) 